\title{
On the Usefulness of Cooperation in $N$ Person Games
}

\author{
Mikhail Sergeevich Nikolskii ${ }^{1}$ and Aboubacar Moussa ${ }^{2}$ \\ ${ }^{1}$ Steklov Mathematical Institute, Russian Academy of Sciences, Gubkina Str. 8, Moscow 119991, Russia \\ ${ }^{2}$ Department of Mathematics and Computer Science, Faculty of Sciences, Abdou Moumouni University, BP 10662, Niamey, Niger
}

Correspondence should be addressed to Aboubacar Moussa; msaboubacar@yahoo.fr

Received 28 June 2016; Revised 11 September 2016; Accepted 26 September 2016

Academic Editor: Bo Yu

Copyright (C) 2016 M. S. Nikolskii and A. Moussa. This is an open access article distributed under the Creative Commons Attribution License, which permits unrestricted use, distribution, and reproduction in any medium, provided the original work is properly cited.

\begin{abstract}
The $N$ person games in which each player maximizes his payoff function are considered. We have studied an interesting question for the cooperative game theory about the usefulness of uniting the $N$ players in a union. The aim of such cooperation is for each player to get a positive increase to his guaranteed payoff. We have obtained some effective sufficient conditions under which the joining of the players in union is useful for each player. The linear case, specially, is being considered. In the second part of the paper, we have studied the question about the usefulness of cooperation of the $N$ players in the presence of the $(N+1)$ th player, an ill-intentioned destructive player, whose whole aim is not to win but to harm each player individually, and also the union of these players, for example, global terrorism. It should be noted that the considered situation in the second part is related to A. V. Kryazhimskiy's talk delivered in the summer of 2014. We obtain constructive conditions under which the union of the players is beneficial in this situation as well.
\end{abstract}

\section{Introduction}

In the game theory (see, e.g., [1-3]), much attention is given to the cooperative game theory of $N$ person. In $[4,5]$, we considered the games of two and three persons from the point of view of the usefulness of combining them in an alliance in order to get additional dividends. In the first part of this paper, we considered $N$ person games in terms of usefulness of uniting players in union in which the choice of strategies is made in concert in order to maximize the sum of payoffs of the $N$ players. In the second part, $N$ players games with perturbing factors of an ill-intentioned destructive player are considered. Here we studied the feasibility of joining (cooperation) the $N$ players in union to counter the possible troubles from the ill-intentioned destructive player. For the different aspects of the theory of cooperation see, for example, [6-9].

\section{Usefulness of Cooperation in $N$ Person Game}

Let $N \geqslant 3$; in the Euclidean arithmetical spaces $\mathbb{R}^{k_{1}}, \ldots$, $\mathbb{R}^{k_{N}}\left(k_{1} \geqslant 1, \ldots, k_{N} \geqslant 1\right)\left(k_{1}, \ldots, k_{N}\right.$-dimensions of the spaces) fixed nonvoid compact set $X_{1}, \ldots, X_{N}$, respectively, and on $X_{1} \times \cdots \times X_{N}$ we define the continuous scalar functions $f_{1}\left(\mathbf{x}_{1}, \ldots, \mathbf{x}_{N}\right), \ldots, f_{N}\left(\mathbf{x}_{1}, \ldots, \mathbf{x}_{N}\right)$. We denote $I=$ $\{1,2, \ldots, N\}$. In the considered game the $i$ th player chooses the vector $\mathbf{x}_{i} \in X_{i}$ and strives to maximize his payoff $f_{i}\left(\mathbf{x}_{1}, \ldots, \mathbf{x}_{N}\right), i \in I$. The players make the choice of vectors $\mathbf{x}_{1}, \ldots, \mathbf{x}_{N}$ independently of each other. As we know from the game theory (see, e.g., [1-3]) each $i$ th player can guarantee a payoff

$$
\gamma_{i}=\max _{\mathbf{x}_{i}} \min _{\substack{\mathbf{x}_{1}, \ldots, \mathbf{x}_{i-1}, \mathbf{x}_{i+1}, \ldots, \mathbf{x}_{N}}} f_{i}\left(\mathbf{x}_{1}, \ldots, \mathbf{x}_{N}\right), \quad i \in I,
$$

where $\mathbf{x}_{1} \in X_{1}, \ldots, \mathbf{x}_{N} \in X_{N}$ (in this formula, it is considered that if $i=1$ the minimization is carried out over $\mathbf{x}_{2}, \ldots, \mathbf{x}_{N}$ and if $i=N$ the minimization is carried out over $\left.\mathbf{x}_{1}, \ldots, \mathbf{x}_{N-1}\right)$, if he chooses a vector $\mathbf{x}_{i}^{*} \in X_{i}$ according to the requirement

$$
\gamma_{i}=\min _{\substack{\mathbf{x}_{1}, \ldots, \mathbf{x}_{i-1}, \mathbf{x}_{i+1}, \ldots, \mathbf{x}_{N}}} f_{i}\left(\mathbf{x}_{1}, \ldots, \mathbf{x}_{i-1}, \mathbf{x}_{i}^{*}, \mathbf{x}_{i+1}, \ldots, \mathbf{x}_{N}\right), \quad i \in I,
$$

where $\mathbf{x}_{1} \in X_{1}, \ldots, \mathbf{x}_{N} \in X_{N}$ (in this formula, it is considered that if $i=1$ the minimization is carried out over $\mathbf{x}_{2}, \ldots, \mathbf{x}_{N}$ 
for $\mathbf{x}_{1}=\mathbf{x}_{1}^{*}$ and if $i=N$ the minimization is carried out over $\mathbf{x}_{1}, \ldots, \mathbf{x}_{N-1}$ for $\mathbf{x}_{N}=\mathbf{x}_{N}^{*}$ ). It is assumed that the payoffs $f_{i}\left(\mathbf{x}_{1}, \ldots, \mathbf{x}_{N}\right), i \in I$, are measured in the same physical units (in economic applications, e.g., payoffs $f_{i}\left(\mathbf{x}_{1}, \ldots, \mathbf{x}_{N}\right)$, $i \in I$, are usually measured in monetary units). In this kind of assumption, the value

$$
\sum_{i=1}^{N} f_{i}\left(\mathbf{x}_{1}, \ldots, \mathbf{x}_{N}\right)
$$

has also a physical sense. If $N$ players are joined together in a union (coalition), then acting concertedly (i.e., choosing $\left(\mathbf{x}_{1}, \ldots, \mathbf{x}_{N}\right)$ in $\left.X_{1} \times \cdots \times X_{N}\right)$ they can use the quantity

$$
\gamma_{N+1}=\max _{\mathbf{x}_{1}, \ldots, \mathbf{x}_{N}}\left(\sum_{i=1}^{N} f_{i}\left(\mathbf{x}_{1}, \ldots, \mathbf{x}_{N}\right)\right),
$$

where $\mathbf{x}_{1} \in X_{1}, \ldots, \mathbf{x}_{N} \in X_{N}$. It is easy to prove that (see (1))

$$
\gamma_{N+1} \geqslant \gamma_{1}+\gamma_{2}+\cdots+\gamma_{N}
$$

If

$$
\gamma_{N+1}>\gamma_{1}+\gamma_{2}+\cdots+\gamma_{N}
$$

then the joining of the players in a union (coalition) is advantageous to all players, since the positive quantity

$$
\Delta=\gamma_{N+1}-\left(\gamma_{1}+\gamma_{2}+\cdots+\gamma_{N}\right)
$$

can be distributed in the form of positive addition to guaranteed payoffs $\gamma_{1}, \gamma_{2}, \ldots, \gamma_{N}$. To know how actually this distribution is reasonable to do, see, for example, [10, c. 181186].

There arises an interesting question for the game theory and its applications of finding constructive conditions on the elements of the game considered by us under which strict inequality (6) holds. We consider two cases, in which it is possible to indicate such conditions.

2.1. Case A. The continuous functions $f_{1}, f_{2}, \ldots, f_{N}$ have on $X_{1} \times X_{2} \times \cdots \times X_{N}$ a separated form:

$$
\begin{aligned}
& f_{1}\left(\mathbf{x}_{1}, \mathbf{x}_{2}, \ldots, \mathbf{x}_{N}\right)=f_{11}\left(\mathbf{x}_{1}\right)+f_{12}\left(\mathbf{x}_{2}\right)+\cdots \\
& \quad+f_{1 N}\left(\mathbf{x}_{N}\right), \\
& f_{2}\left(\mathbf{x}_{1}, \mathbf{x}_{2}, \ldots, \mathbf{x}_{N}\right)=f_{21}\left(\mathbf{x}_{1}\right)+f_{22}\left(\mathbf{x}_{2}\right)+\cdots \\
& \quad+f_{2 N}\left(\mathbf{x}_{N}\right), \\
& \quad \vdots \\
& f_{N}\left(\mathbf{x}_{1}, \mathbf{x}_{2}, \ldots, \mathbf{x}_{N}\right)=f_{N 1}\left(\mathbf{x}_{1}\right)+f_{N 2}\left(\mathbf{x}_{2}\right)+\cdots \\
& \quad+f_{N N}\left(\mathbf{x}_{N}\right),
\end{aligned}
$$

where the functions $f_{1 i}\left(\mathbf{x}_{i}\right), f_{2 i}\left(\mathbf{x}_{i}\right), \ldots, f_{N i}\left(\mathbf{x}_{i}\right)$ are continuous on $X_{i}, i \in I$.
In what follows, let us agree to write operations $\max _{\mathbf{x}_{1}}, \ldots$, $\max _{\mathbf{x}_{N}}$ instead of the operations $\max _{\mathbf{x}_{1} \in X_{1}}, \ldots, \max _{\mathbf{x}_{N} \in X_{N}}$, respectively. Similarly, we write $\min _{\mathbf{x}_{1}}, \ldots, \min _{\mathbf{x}_{N}}$ instead of the operations $\min _{\mathbf{x}_{1} \in X_{1}}, \ldots, \min _{\mathbf{x}_{N} \in X_{N}}$, respectively. Investigated inequality (6) can be rewritten as

$$
\begin{aligned}
\max _{\mathbf{x}_{1}} & {\left[f_{11}\left(\mathbf{x}_{1}\right)+f_{21}\left(\mathbf{x}_{1}\right)+\cdots+f_{N 1}\left(\mathbf{x}_{1}\right)\right] } \\
+ & \max _{\mathbf{x}_{2}}\left[f_{12}\left(\mathbf{x}_{2}\right)+f_{22}\left(\mathbf{x}_{2}\right)+\cdots+f_{N 2}\left(\mathbf{x}_{2}\right)\right]+\cdots \\
+ & \max _{\mathbf{x}_{N}}\left[f_{1 N}\left(\mathbf{x}_{N}\right)+f_{2 N}\left(\mathbf{x}_{N}\right)+\cdots+f_{N N}\left(\mathbf{x}_{N}\right)\right] \\
& >\left(\max _{\mathbf{x}_{1}} f_{11}\left(\mathbf{x}_{1}\right)+\min _{\mathbf{x}_{1}} f_{21}\left(\mathbf{x}_{1}\right)+\cdots\right. \\
& \left.+\min _{\mathbf{x}_{1}} f_{N 1}\left(\mathbf{x}_{1}\right)\right)+\left(\max _{\mathbf{x}_{2}} f_{22}\left(\mathbf{x}_{2}\right)+\min _{\mathbf{x}_{2}} f_{12}\left(\mathbf{x}_{2}\right)\right. \\
& \left.+\min _{\mathbf{x}_{2}} f_{32}\left(\mathbf{x}_{2}\right)+\cdots+\min _{\mathbf{x}_{2}} f_{N 2}\left(\mathbf{x}_{2}\right)\right)+\cdots \\
& +\left(\max _{\mathbf{x}_{N}} f_{N N}\left(\mathbf{x}_{N}\right)+\min _{\mathbf{x}_{N}} f_{1 N}\left(\mathbf{x}_{N}\right)+\min _{\mathbf{x}_{N}} f_{2 N}\left(\mathbf{x}_{N}\right)\right. \\
& \left.+\cdots+\min _{\mathbf{x}_{N}} f_{N-1 N}\left(\mathbf{x}_{N}\right)\right) .
\end{aligned}
$$

We consider separately for $i \in I$ the inequality

$$
\begin{aligned}
\max _{\mathbf{x}_{i}}[ & \left.f_{1 i}\left(\mathbf{x}_{i}\right)+f_{2 i}\left(\mathbf{x}_{i}\right)+\cdots+f_{N i}\left(\mathbf{x}_{i}\right)\right] \\
\geqslant & \max _{\mathbf{x}_{i}} f_{i i}\left(\mathbf{x}_{i}\right)+\min _{\mathbf{x}_{i}} f_{1 i}\left(\mathbf{x}_{i}\right)+\cdots+\min _{\mathbf{x}_{i}} f_{i-1 i}\left(\mathbf{x}_{i}\right) \\
& \quad+\min _{\mathbf{x}_{i}} f_{i+1 i}\left(\mathbf{x}_{i}\right)+\cdots+\min _{\mathbf{x}_{i}} f_{N i}\left(\mathbf{x}_{i}\right) .
\end{aligned}
$$

In this formula, it is considered that the right-hand side if $i=$ 1 has the form

$$
\max _{\mathbf{x}_{1}} f_{11}\left(\mathbf{x}_{1}\right)+\min _{\mathbf{x}_{1}} f_{21}\left(\mathbf{x}_{1}\right)+\cdots+\min _{\mathbf{x}_{1}} f_{N 1}\left(\mathbf{x}_{1}\right)
$$

and if $i=N$ has the form

$$
\begin{aligned}
& \max _{\mathbf{x}_{N}} f_{N N}\left(\mathbf{x}_{N}\right)+\min _{\mathbf{x}_{N}} f_{1 N}\left(\mathbf{x}_{N}\right)+\cdots \\
& \quad+\min _{\mathbf{x}_{N}} f_{N-1 N}\left(\mathbf{x}_{N}\right) .
\end{aligned}
$$

It is easy to prove the following lemma.

Lemma 1. Under assumptions (8) and (9) for each $i \in I$ inequality (10) holds.

Note that, in general, for each $i \in I$ inequality (10) is not necessarily fulfilled strictly. However one has the following.

Lemma 2. For a given $i \in I$, there exists a point $\mathbf{x}_{i}^{*}$ of the set $\arg \max _{\mathbf{x}_{i}} f_{i i}\left(\mathbf{x}_{i}\right)$, which does not belong to at least one of the sets arg $\min _{\mathbf{x}_{i}} f_{j i}\left(\mathbf{x}_{i}\right)$, where $j \in I$ and is not equal to $i$. Then inequality (10) holds in the strict sense.

Proof. It is evident that for a given $i \in I$ the left-hand side of inequality (10) for $\mathbf{x}_{i}=\mathbf{x}_{i}^{*}$ is greater than or equal to the value

$$
f_{1 i}\left(\mathbf{x}_{i}^{*}\right)+f_{2 i}\left(\mathbf{x}_{i}^{*}\right)+\cdots+f_{N i}\left(\mathbf{x}_{i}^{*}\right),
$$


where it is not difficult to see that it is greater than or equal to the right side of inequality (10). Further, by using the hypotheses of Lemma 2, it is easy to prove that this value is strictly greater than the right-hand side of inequality (10). From what has been said, Lemma 2 follows.

Remark 3. The symbols $\arg \max _{\mathbf{x}_{i}} \omega_{j i}\left(\mathbf{x}_{i}\right)$ and $\arg \min _{\mathbf{x}_{i}} \omega_{j i}\left(\mathbf{x}_{i}\right)$ denote, respectively, the sets of points of maximum and minimum of the function $\omega_{j i}\left(\mathbf{x}_{i}\right)$ on $X_{i}$.

From the above we get the following.

Theorem 4. If at least for one number $i \in I$ the conditions of Lemma 2 are realized, then one has strict inequality (9) and, therefore, strict inequality (6).

2.2. Case $B$ (Subcase of Case $A$ ). The sets $X_{1}, X_{2}, \ldots, X_{N}$, convex compact, and the continuous functions $f_{1}\left(\mathbf{x}_{1}, \ldots, \mathbf{x}_{N}\right)$, $f_{2}\left(\mathbf{x}_{1}, \ldots, \mathbf{x}_{N}\right), \ldots, f_{N}\left(\mathbf{x}_{1}, \ldots, \mathbf{x}_{N}\right)$ have on $X_{1} \times X_{2} \times \cdots \times X_{N}$ linear form

$$
\begin{aligned}
& f_{1}\left(\mathbf{x}_{1}, \ldots, \mathbf{x}_{N}\right)=\left\langle\mathbf{a}_{11}, \mathbf{x}_{1}\right\rangle+\left\langle\mathbf{a}_{12}, \mathbf{x}_{2}\right\rangle+\cdots \\
& +\left\langle\mathbf{a}_{1 N}, \mathbf{x}_{N}\right\rangle, \\
& f_{2}\left(\mathbf{x}_{1}, \ldots, \mathbf{x}_{N}\right)=\left\langle\mathbf{a}_{21}, \mathbf{x}_{1}\right\rangle+\left\langle\mathbf{a}_{22}, \mathbf{x}_{2}\right\rangle+\cdots \\
& +\left\langle\mathbf{a}_{2 N}, \mathbf{x}_{N}\right\rangle, \\
& \vdots \\
& f_{N}\left(\mathbf{x}_{1}, \ldots, \mathbf{x}_{N}\right)=\left\langle\mathbf{a}_{N 1}, \mathbf{x}_{1}\right\rangle+\left\langle\mathbf{a}_{N 2}, \mathbf{x}_{2}\right\rangle+\cdots \\
& \quad+\left\langle\mathbf{a}_{N N}, \mathbf{x}_{N}\right\rangle,
\end{aligned}
$$

where $\mathbf{a}_{1 i}, \mathbf{a}_{2 i}, \ldots, \mathbf{a}_{N i}$ are fixed vectors in $\mathbb{R}^{k_{i}}, i \in I$; the symbol $\langle\cdot, \cdot\rangle$ denotes the standard scalar product in $\mathbb{R}^{k_{1}}, \ldots, \mathbb{R}^{k_{N}}$, respectively. As the functions $f_{1}\left(\mathbf{x}_{1}, \ldots, \mathbf{x}_{N}\right)$, $f_{2}\left(\mathbf{x}_{1}, \ldots, \mathbf{x}_{N}\right), \ldots, f_{N}\left(\mathbf{x}_{1}, \ldots, \mathbf{x}_{N}\right)$ have a separated form (cf. (8)), you can use the results that we obtained in Case A. In considered linear Case B, strict inequality (10) can be rewritten for each $i \in I$ in the form

$$
\begin{aligned}
\max _{\mathbf{x}_{i}} & \left(\left\langle\mathbf{a}_{1 i}+\mathbf{a}_{2 i}+\cdots+\mathbf{a}_{N i}, \mathbf{x}_{i}\right\rangle\right) \\
> & \max _{\mathbf{x}_{i}}\left\langle\mathbf{a}_{i i}, \mathbf{x}_{i}\right\rangle+\min _{\mathbf{x}_{i}}\left\langle\mathbf{a}_{1 i}, \mathbf{x}_{i}\right\rangle+\min _{\mathbf{x}_{i}}\left\langle\mathbf{a}_{2 i}, \mathbf{x}_{i}\right\rangle+\cdots \\
& +\min _{\mathbf{x}_{i}}\left\langle\mathbf{a}_{i-1 i}, \mathbf{x}_{i}\right\rangle+\min _{\mathbf{x}_{i}}\left\langle\mathbf{a}_{i+1 i}, \mathbf{x}_{i}\right\rangle+\cdots \\
& +\min _{\mathbf{x}_{i}}\left\langle\mathbf{a}_{N i}, \mathbf{x}_{i}\right\rangle .
\end{aligned}
$$

In what follows we need some auxiliary information.

In the Euclidean space $\mathbb{R}^{m}(m \geqslant 1)$ we denote $\sigma_{m}=\{\mathbf{v} \epsilon$ $\left.\mathbb{R}^{m}:|\mathbf{v}|=1\right\}$, where $|\mathbf{v}|$ mean standard length of the vector $\mathbf{v}$. The following will be useful.

Definition 5. A nonvoid convex compact set $K \subset \mathbb{R}^{m}(m \geqslant 2)$ with nonvoid interior is called an $S$-set, if

(1) for any $\psi \in \sigma_{m}$ in $K$ there exists only one vector $\mathbf{v}(\psi)$, maximizing on $\mathbf{v} \in K$ the scalar product $\langle\mathbf{v}, \psi\rangle$;
(2) for each boundary point $\mathbf{v}_{0}$ of the compact set $K$ there exists only one support hyperplane, passing through the point $\mathbf{v}_{0}$.

Remark 6. Here and further, we use some notions of convex analysis (see, e.g., [11, 12]). We note that for each $\psi \in \sigma_{m}$ the point $\mathbf{v}(\psi)$ belongs to the boundary of the set $K$. Using the terminology of convex analysis, we can say that the $S$-set is a strictly convex set and also a convex body.

In [4] is proved the following.

Lemma 7. Let the set $K \subset \mathbb{R}^{m}(m \geqslant 2)$ be an S-set, and let vectors $\mathbf{p}$ and $\mathbf{q}$ be some nonzero vectors in $\mathbb{R}^{m}$, such that

$$
\frac{1}{|\mathbf{p}|} \mathbf{p} \neq \frac{1}{|\mathbf{q}|}(-\mathbf{q})
$$

Then,

$$
(\langle\mathbf{v}(\mathbf{p}),-\mathbf{q}\rangle)<(\langle\mathbf{v}(-\mathbf{q}),-\mathbf{q}\rangle) .
$$

Lemma 8. For a given $i \in I$, the set $X_{i} \subset \mathbb{R}^{k_{i}}\left(k_{i} \geqslant 2\right)$ is an $S$ set, and the vectors $\mathbf{a}_{1 i}, \mathbf{a}_{2 i}, \ldots, \mathbf{a}_{N i}$ are nonzero and the vector $\left(1 /\left|\mathbf{a}_{i i}\right|\right) \mathbf{a}_{i i}$ is not equal to at least one of the vectors

$$
\frac{1}{\left|\mathbf{a}_{j i}\right|}\left(-\mathbf{a}_{j i}\right) \text {, }
$$

where $j \in I$ and $j$ is not equal to $i$. Then for this number $i$ one has strict inequality (15).

Proof. Under the above assumptions, for a given $i \in I$, the vector $\mathbf{x}_{i}\left(\mathbf{a}_{i i}\right)$ that is maximizing the function $\left\langle\mathbf{x}_{i}, \mathbf{a}_{i i}\right\rangle$ on $\mathbf{x}_{i} \in$ $X_{i}$ is uniquely defined. Thus,

$$
\underset{\mathbf{x}_{i}}{\arg \max }\left\langle\mathbf{x}_{i}, \mathbf{a}_{i i}\right\rangle=\left\{\mathbf{x}_{i}\left(\mathbf{a}_{i i}\right)\right\} .
$$

Let the vector $\left(1 /\left|\mathbf{a}_{i i}\right|\right) \mathbf{a}_{i i}$ be not equal to the vector $\left(1 /\left|\mathbf{a}_{k i}\right|\right)\left(-\mathbf{a}_{k i}\right)$, where $k \in I$ and $k$ is not equal to $i$. From Lemma 7 we obtain the following inequality:

$$
\left(\left\langle\mathbf{x}_{i}\left(\mathbf{a}_{i i}\right),-\mathbf{a}_{k i}\right\rangle\right)<\left(\left\langle\mathbf{x}_{i}\left(-\mathbf{a}_{k i}\right),-\mathbf{a}_{k i}\right\rangle\right),
$$

where $\mathbf{x}_{i}\left(-\mathbf{a}_{k i}\right)$ means the maximizer of the function $\left\langle\mathbf{x}_{i},-\mathbf{a}_{k i}\right\rangle$ for $\mathbf{x}_{i} \in X_{i}$.

We can show that

$$
\min _{\mathbf{x}_{i}}\left\langle\mathbf{x}_{i}, \mathbf{a}_{k i}\right\rangle=-\left\langle\mathbf{x}_{i}\left(-\mathbf{a}_{k i}\right),-\mathbf{a}_{k i}\right\rangle .
$$

From relations (20) and (21) we obtain the inequality

$$
\left(\left\langle\mathbf{x}_{i}\left(\mathbf{a}_{i i}\right), \mathbf{a}_{k i}\right\rangle\right)>\min _{\mathbf{x}_{i}}\left\langle\mathbf{x}_{i}, \mathbf{a}_{k i}\right\rangle .
$$

Hence it follows that

$$
\mathbf{x}_{i}\left(\mathbf{a}_{i i}\right) \notin \underset{\mathbf{x}_{i}}{\arg \min }\left\langle\mathbf{x}_{i}, \mathbf{a}_{k i}\right\rangle .
$$

From the above and Lemma 2, it follows that strict inequality (15) holds. 
On the basis of the above, we obtain the following theorem.

Theorem 9. If for at least one number $i \in I$ the conditions of Lemma 8 are realized, then for the functions $f_{j}\left(\mathbf{x}_{1}, \ldots, \mathbf{x}_{N}\right), j=$ $1, \ldots N$, one has strict inequality (9) and hence strict inequality (6).

Remark 10. The payoff functions, having a separated form (see (8)), are often considered in the game theory (see, e.g., [10]).

\section{Usefulness of Cooperation in $N$ Person Game in Presence of an Ill-Intentioned Destructive Player}

In this part, we briefly studied some more general game models than in the first part which considers the existence of the $(N+1)$ th player, an ill-intentioned destructive player. The statement of the considered problem here was motivated by A. V. Kryazhimskiy's talk.

We consider the $N$ person game in a form almost classical, where $N \geqslant 3$. The payoff function of the $i$ th player is a continuous function

$$
f_{i}\left(\mathbf{x}_{1}, \ldots, \mathbf{x}_{N}, \mathbf{y}\right), \quad i \in I
$$

where $\mathbf{x}_{1} \in X_{1}, \ldots, \mathbf{x}_{N} \in X_{N}, \mathbf{y} \in Y$ and $X_{1}, \ldots, X_{N}$, $Y$ are nonvoid compact sets in the corresponding finite dimensional Euclidean spaces. The $i$ th player selects $\mathbf{x}_{i} \in X_{i}$ within order to maximize

$$
f_{i}\left(\mathbf{x}_{1}, \ldots, \mathbf{x}_{N}, \mathbf{y}\right), \quad i \in I
$$

The vector $y \in Y$ is selected by the ill-intentioned destructive player, whose objectives are not to win, but to harm the players. We want to prove that sometimes it is advantageous for the players to be united in a coalition and fight together with the possible actions of the ill-intentioned destructive player.

We will study the game, for which

$$
\begin{aligned}
\max _{\mathbf{x}_{1}, \ldots, \mathbf{x}_{N}} & \min _{\mathbf{y}}\left(\sum_{i=1}^{N} f_{i}\left(\mathbf{x}_{1}, \ldots, \mathbf{x}_{N}, \mathbf{y}\right)\right) \\
> & \max _{\mathbf{x}_{1}} \min _{\mathbf{x}_{2}, \ldots, \mathbf{x}_{N}, \mathbf{y}} f_{1}\left(\mathbf{x}_{1}, \ldots, \mathbf{x}_{N}, \mathbf{y}\right) \\
& +\max _{\mathbf{x}_{2}} \min _{\mathbf{x}_{1}, \mathbf{x}_{3}, \ldots, \mathbf{x}_{N}, \mathbf{y}} f_{2}\left(\mathbf{x}_{1}, \ldots, \mathbf{x}_{N}, \mathbf{y}\right)+\cdots \\
& +\max _{\mathbf{x}_{N}} \min _{\mathbf{x}_{1}, \ldots, \mathbf{x}_{N-1}, \mathbf{y}} f_{N}\left(\mathbf{x}_{1}, \ldots, \mathbf{x}_{N}, \mathbf{y}\right) .
\end{aligned}
$$

Here and further in similar inequalities $\mathbf{x}_{1} \in X_{1}, \ldots, \mathbf{x}_{N} \in$ $X_{N}, \mathbf{y} \in Y$.

Note that when inequality (26) is fulfilled, it is advantageous for the players to be united in a coalition in order to get greater payoff through bargaining than in a two-person zero-sum game. In fact, in inequality (26) we use the notion of the characteristic function from cooperative game theory. Our study is greatly simplified if

$$
\begin{aligned}
& f_{1}\left(\mathbf{x}_{1}, \ldots, \mathbf{x}_{N}, \mathbf{y}\right)=f_{11}\left(\mathbf{x}_{1}, \ldots, \mathbf{x}_{N}\right)+f_{12}(\mathbf{y}), \\
& f_{2}\left(\mathbf{x}_{1}, \ldots, \mathbf{x}_{N}, \mathbf{y}\right)=f_{21}\left(\mathbf{x}_{1}, \ldots, \mathbf{x}_{N}\right)+f_{22}(\mathbf{y}),
\end{aligned}
$$

$$
f_{N}\left(\mathbf{x}_{1}, \ldots, \mathbf{x}_{N}, \mathbf{y}\right)=f_{N 1}\left(\mathbf{x}_{1}, \ldots, \mathbf{x}_{N}\right)+f_{N 2}(\mathbf{y}),
$$

where the functions $f_{11}, f_{21}, \ldots, f_{N 1}$ are continuous on $X_{1} \times$ $X_{2} \times \cdots \times X_{N}$, while the functions $f_{12}, f_{22}, \ldots, f_{N 2}$ are continuous on $Y$.

By using (27) relation (26) can be rewritten in the form

$$
\begin{aligned}
& \max _{\mathbf{x}_{1}, \ldots, \mathbf{x}_{N}}\left[f_{11}\left(\mathbf{x}_{1}, \ldots, \mathbf{x}_{N}\right)+f_{21}\left(\mathbf{x}_{1}, \ldots, \mathbf{x}_{N}\right)+\cdots\right. \\
& \left.\quad+f_{N 1}\left(\mathbf{x}_{1}, \ldots, \mathbf{x}_{N}\right)\right]+\min _{\mathbf{y}}\left[f_{12}(\mathbf{y})+f_{22}(\mathbf{y})+\cdots\right. \\
& \left.\quad+f_{N 2}(\mathbf{y})\right]>\max _{\mathbf{x}_{1}} \min _{\mathbf{x}_{2}, \mathbf{x}_{3}, \ldots, \mathbf{x}_{N}} f_{11}\left(\mathbf{x}_{1}, \ldots, \mathbf{x}_{N}\right) \\
& \quad+\max _{\mathbf{x}_{2}} \min _{\mathbf{x}_{1}, \mathbf{x}_{3}, \ldots, \mathbf{x}_{N}} f_{21}\left(\mathbf{x}_{1}, \ldots, \mathbf{x}_{N}\right)+\cdots \\
& \quad+\max _{\mathbf{x}_{N}} \min _{\mathbf{x}_{1}, \ldots, \mathbf{x}_{N-1}} f_{N 1}\left(\mathbf{x}_{1}, \ldots, \mathbf{x}_{N}\right)+\min _{\mathbf{y}} f_{12}(\mathbf{y}) \\
& \quad+\min _{\mathbf{y}} f_{22}(\mathbf{y})+\cdots+\min _{\mathbf{y}} f_{N 2}(\mathbf{y}) .
\end{aligned}
$$

Note that the following inequality holds:

$$
\begin{aligned}
& \max _{\mathbf{x}_{1}, \ldots, \mathbf{x}_{N}}\left[f_{11}\left(\mathbf{x}_{1}, \ldots, \mathbf{x}_{N}\right)+f_{21}\left(\mathbf{x}_{1}, \ldots, \mathbf{x}_{N}\right)+\cdots\right. \\
& \left.\quad+f_{N 1}\left(\mathbf{x}_{1}, \ldots, \mathbf{x}_{N}\right)\right] \\
& \quad \geqslant \max _{\mathbf{x}_{1}} \min _{\mathbf{x}_{2}, \mathbf{x}_{3}, \ldots, \mathbf{x}_{N}} f_{11}\left(\mathbf{x}_{1}, \ldots, \mathbf{x}_{N}\right) \\
& \quad+\max _{\mathbf{x}_{2}} \min _{\mathbf{x}_{1}, \mathbf{x}_{3}, \ldots, \mathbf{x}_{N}} f_{21}\left(\mathbf{x}_{1}, \ldots, \mathbf{x}_{N}\right)+\cdots \\
& \quad+\max _{\mathbf{x}_{N}} \min _{\mathbf{x}_{1}, \ldots, \mathbf{x}_{N-1}} f_{N 1}\left(\mathbf{x}_{1}, \ldots, \mathbf{x}_{N}\right) .
\end{aligned}
$$

Proof of Inequality (29). We have

$$
\begin{aligned}
f_{11}\left(\mathbf{x}_{1}, \ldots, \mathbf{x}_{N}\right)+f_{21}\left(\mathbf{x}_{1}, \ldots, \mathbf{x}_{N}\right)+\cdots \\
\quad+f_{N 1}\left(\mathbf{x}_{1}, \ldots, \mathbf{x}_{N}\right) \\
\geqslant \min _{\mathbf{x}_{2}, \mathbf{x}_{3}, \ldots, \mathbf{x}_{N}} f_{11}\left(\mathbf{x}_{1}, \ldots, \mathbf{x}_{N}\right) \\
\quad+\min _{\mathbf{x}_{1}, \mathbf{x}_{3}, \ldots, \mathbf{x}_{N}} f_{21}\left(\mathbf{x}_{1}, \ldots, \mathbf{x}_{N}\right)+\cdots \\
\quad+\min _{\mathbf{x}_{1}, \ldots, \mathbf{x}_{N-1}} f_{N 1}\left(\mathbf{x}_{1}, \ldots, \mathbf{x}_{N}\right) .
\end{aligned}
$$

Applying to both parts of the operation $\max _{\mathbf{x}_{1}, \mathbf{x}_{2}, \ldots, \mathbf{x}_{N}}$ we obtain the required inequality.

From (29) it follows that for the fulfillment of inequality (28) it is sufficient to guarantee the inequality

$$
\begin{aligned}
\min _{\mathbf{y}} & {\left[f_{12}(\mathbf{y})+f_{22}(\mathbf{y})+\cdots+f_{N 2}(\mathbf{y})\right] } \\
> & \min _{\mathbf{y}} f_{12}(\mathbf{y})+\min _{\mathbf{y}} f_{22}(\mathbf{y})+\cdots+\min _{\mathbf{y}} f_{N 2}(\mathbf{y}) .
\end{aligned}
$$


Note that in the general case

$$
\begin{aligned}
\min _{\mathbf{y}} & {\left[f_{12}(\mathbf{y})+f_{22}(\mathbf{y})+\cdots+f_{N 2}(\mathbf{y})\right] } \\
\geqslant & \min _{\mathbf{y}} f_{12}(\mathbf{y})+\min _{\mathbf{y}} f_{22}(\mathbf{y})+\cdots+\min _{\mathbf{y}} f_{N 2}(\mathbf{y}) .
\end{aligned}
$$

Therefore, for inequality (31) to be realized, it is necessary that inequality (32) must become strict.

It is proved by contradiction that strict inequality (31) holds if

$$
\begin{gathered}
\underset{\mathbf{y} \in Y}{\arg \min } f_{12}(\mathbf{y}) \cap \underset{\mathbf{y} \in Y}{\arg \min } f_{22}(\mathbf{y}) \cap \cdots \\
\cap \underset{\mathbf{y} \in Y}{\arg \min } f_{N 2}(\mathbf{y})=\varnothing .
\end{gathered}
$$

Note that the fulfillment of condition (33) is independent of the choice of the functions $f_{11}\left(\mathbf{x}_{1}, \ldots, \mathbf{x}_{N}\right)$, $f_{21}\left(\mathbf{x}_{1}, \ldots, \mathbf{x}_{N}\right), \ldots, f_{N 1}\left(\mathbf{x}_{1}, \ldots, \mathbf{x}_{N}\right)$. Thus, it follows from the above that, for strict inequality (28) to be fulfilled, it is sufficient that relation (33) is fulfilled.

\section{Conclusion}

This article relates to the theme of the theory of $N$ person games. In this theory, a distinction of noncooperative and cooperative games is made. The formulations of considered problems are related to the cooperative games theory (see, e.g., [1-3]). Note that the problems we are studying are directly related to the study of the properties of the characteristic function of $N$ person cooperative games theory, where all the players formed a union. The specific participants of the union may be firms, states, and so forth. In the article we have considered the general case of nonlinear payoff functions in the separable form and a subcase of these functions in the linear form. Effective sufficient conditions which ensure the usefulness of the union of all the players in coalition have been obtained. A method of studying $(N+1)$-person games has also been proposed in which $N$ players are active and the $(N+1)$ th player is an ill-intentioned destructive player, whose aim is not to win, but to harm others.

\section{Competing Interests}

The authors declare that there is no conflict of interests regarding the publication of this paper.

\section{Acknowledgments}

The authors would like to thank Professor V. I. Zhukovskii for his valuable comments and suggestions, which improve the presentation of this paper.

\section{References}

[1] J. von Neumann and O. Morgenstern, Theory of Games and Economic Behavior, Princeton University Press, Princeton, NJ, USA, 1953.
[2] L. A. Petrosjan and N. A. Zenkevich, Game Theory, World Scientific, River Edge, NJ, USA, 2nd edition, 2016.

[3] G. Owen, Game Theory, Emerald Group Publishing Limited, Bingley, UK, 4th edition, 2013.

[4] M. S. Nikolskii and M. Aboubacar, "On the benefit of cooperation in two-person games," Mathematical Education, vol. 71, no. 3, pp. 34-40, 2014 (Russian).

[5] M. S. Nikolskii and M. Aboubacar, "On the benefit of cooperation in three-person games," Proceedings of the Steklov Institute of Mathematics, vol. 293, supplement 1, pp. 148-155, 2016.

[6] J. F. Nash, “The bargaining problem," Econometrica, vol. 18, pp. 155-162, 1950.

[7] S. Hart and A. Mas-Colell, "Bargaining and cooperation in strategic form games," Journal of the European Economic Association, vol. 8, no. 1, pp. 7-33, 2010.

[8] A. Okada, "The possibility of cooperation in an n-person prisoners' dilemma with institutional arrangements," Public Choice, vol. 77, no. 3, pp. 629-656, 1993.

[9] H. W. Stuart, "Cooperative games and business strategy," in Game Theory and Business Applications, K. Chatterjee and W. F. Samuelson, Eds., pp. 189-211, Kluwer Academic, Boston, Mass, USA, 2001.

[10] V. I. Zhukovskii, Cooperative Games under Uncertainty and Their Applications, URSS, Moscow, Russia, 2nd edition, 2010 (Russian).

[11] T. Bonnesen and W. Fenchel, Theory of Convex Bodies, BCS Associates, Moscow, Russia, 1987, (Translated from German).

[12] R. T. Rockafellar, Convex Analysis, Princeton University Press, Princeton, NJ, USA, 1970. 


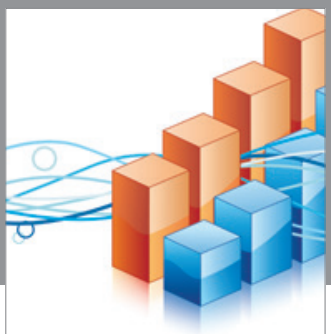

Advances in

Operations Research

vatem alat4

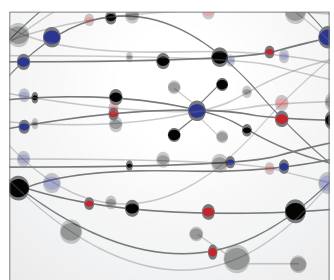

\section{The Scientific} World Journal
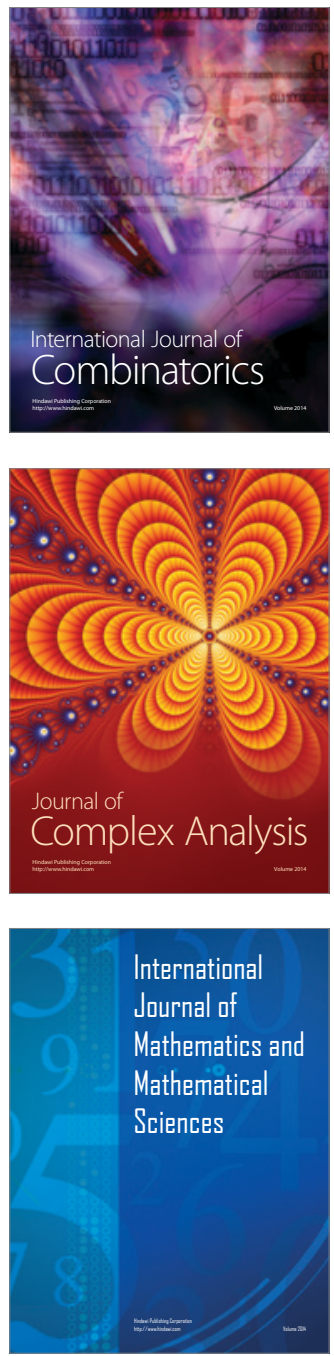
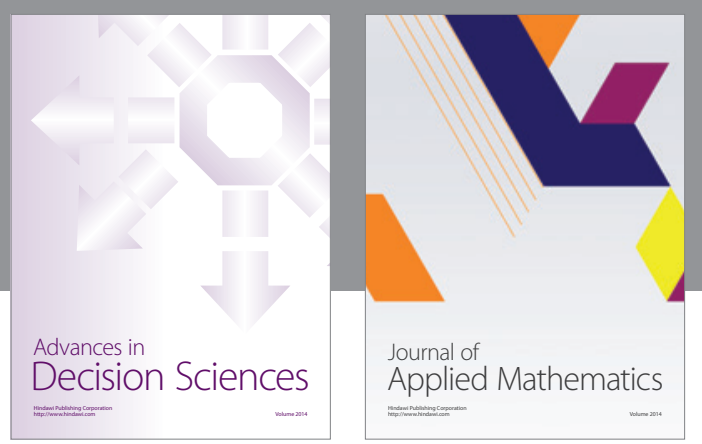

Algebra

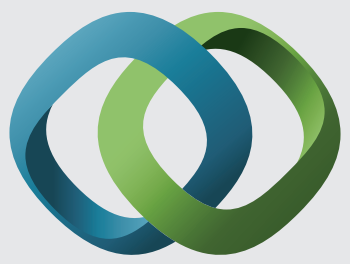

\section{Hindawi}

Submit your manuscripts at

http://www.hindawi.com
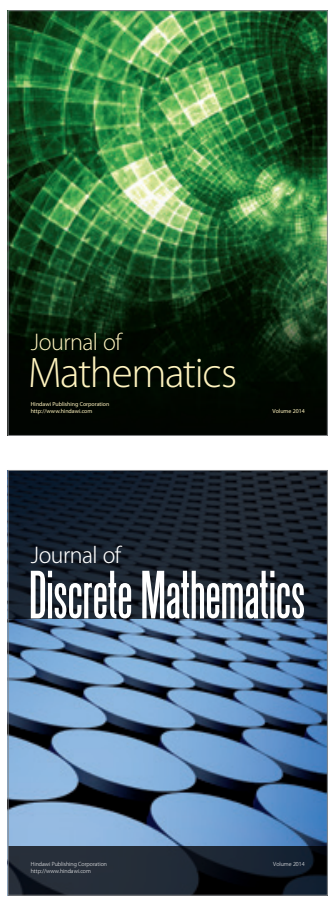

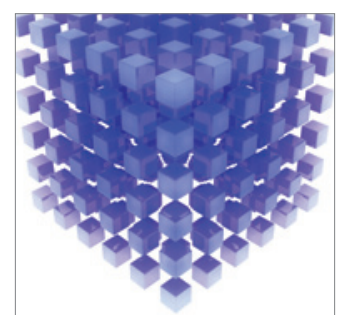

Mathematical Problems in Engineering
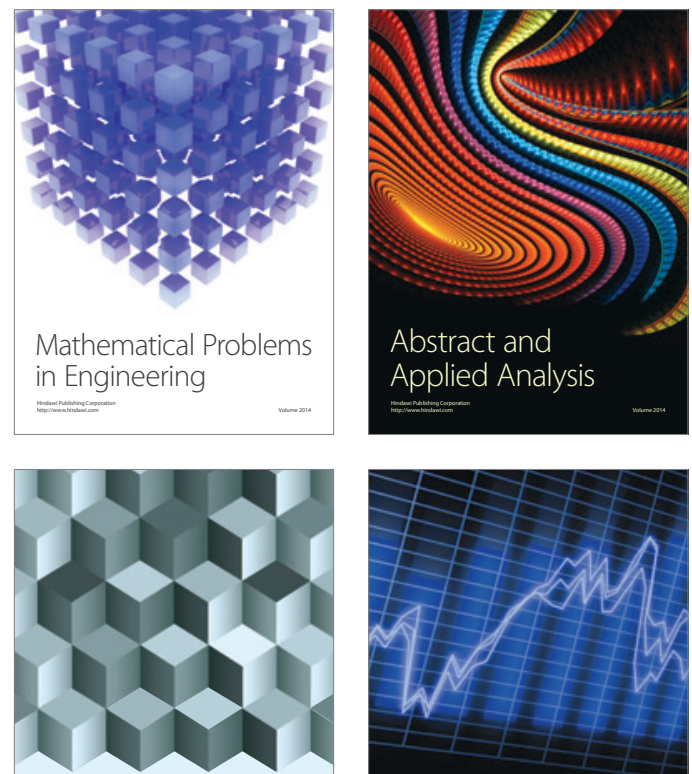

Journal of

Function Spaces

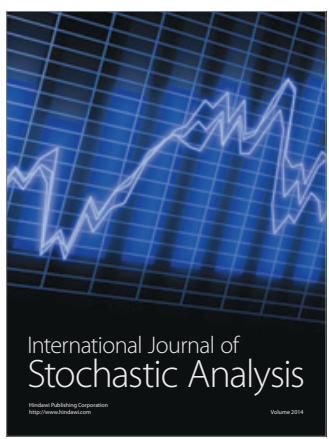

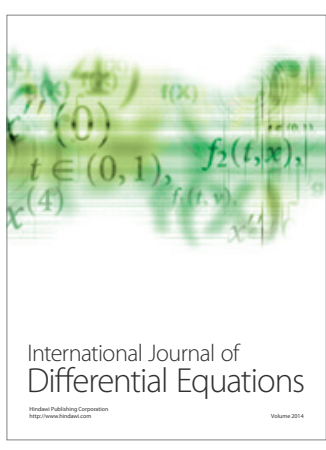
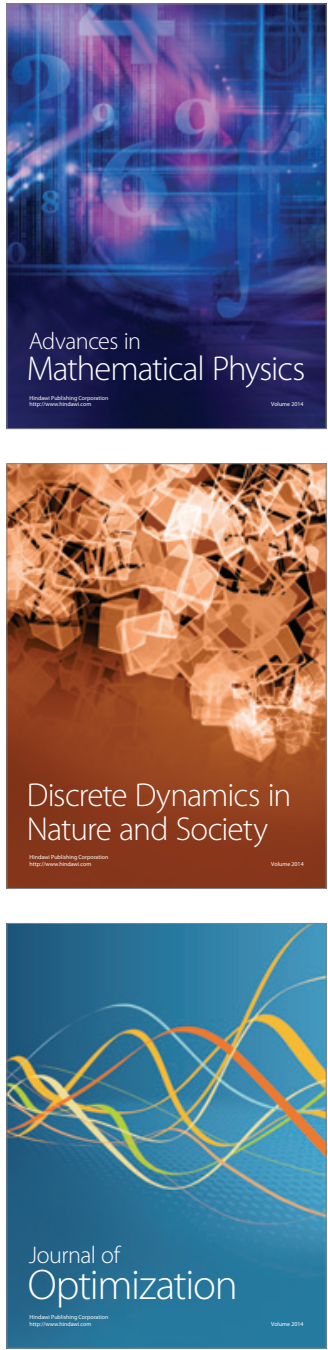